Original Article

Joumal of Epilepsy Research pISSN 2233-6249 / eISSN 2233-6257

Received June 28, 2016

Accepted December 30, 2017

Corresponding author: Manjari Tripathi, DM Department of Neurology, Room No. 705 , Neurosciences Centre, All India Institute of Medical Sciences, New Delhi 110029 , India

Tel. +91-2659-4494, 2658-8248

Fax. $+91-2658-8248,2658-8166$

E-mail; manjari2tripathi@gmail.com

\title{
Predictors of Seizure-Related Injuries in an Epilepsy Cohort from North India
}

Surekha Dabla, DMํㅡ, Inder Puri, DM², Deepa Dash, DM², Padma Madakasira Vasantha, DM², Manjari Tripathi, DM ${ }^{2}$

${ }^{1}$ Department of Medicine, Pt BD sharma, PGIMS Rohtak, Haryana; ${ }^{2}$ Department of Neurology, All India Institute of Medical Sciences, New Delhi, India

Background and Purpose: To identify predictors of seizure-related injury (SRI) and death in people with epilepsy (PWE) in a North Indian cohort.

Methods: This ambispective cohort study included PWE registered in an epilepsy clinic in Delhi between May 2010 and December 2011. Five hundred twenty-six patients were enrolled and followed for 25 months. Patients were categorized into two groups based on SRI/no SRI during the study period. We analyzed various factors to identify predictors of SRI and death.

Results: Of 526 patients, $355(67.5 \%)$ reported having no SRIs and $171(32.5 \%)$ had sustained an SRI. Among patients with SRI, $72.5 \%$ were male; $62 \%$ of those with no SRI were male. The injury type included soft tissue (60\%), head trauma (20\%), dental trauma (10\%), orthopedic (10\%), and burns (5\%). On univariate analysis, factors predicting SRI occurrence were male gender, abnormal birth history $(p<0.01)$, abnormal mental status $(p<0.01)$, seizure duration $(p<0.04)$, daytime seizures $(p<0.05)$, dependence on a caregiver $(p<0.008)$, and uncontrolled seizures $(p<0.001)$, history of cluster seizures or status epilepticus $(p<0.001$ ), occurrence of generalized tonic-clonic seizures (GTCS), and use of $>3$ antiepileptic drugs $(p<0.008)$. On multiple logistic regression analysis, male gender, uncontrolled seizures, history of cluster seizures or status epilepticus, and GTCS were significant risk factors. Sixteen deaths occurred in our cohort, and 13 fit the definition of probable sudden unexpected death in epilepsy (SUDEP). Most patients with SUDEP had an unwitnessed event (69.2\%). The only significant factor in predicting death was uncontrolled seizures.

Conclusions: Male gender, occurrence of GTCS, uncontrolled seizures, and history of cluster seizures or status epilepticus predicted SRI occurrence in PWE. Precautions should be taken by caregivers of patients with these risk factors, to prevent injury. (2018;8:27-32)

Key words: Epilepsy, Injuries, Sudep, Predictors

\section{Introduction}

According to the World Health Organization, epilepsy accounted for about $0.5 \%$ of the global burden of disease in 2005 , or more than 7 million disability-adjusted life-years. Intense clinical research is ongoing to explore various aspects of the risks related to epilepsy. Mortality figures in people with epilepsy (PWE) are about two to three times greater than those in the general population. Most causes of death can be attributed to underlying neurological disease, but some are epilepsy related, including accidents, drowning, status epilepticus, and sudden unexpected death in epilepsy (SUDEP). In a recent pub- lication form Kolkata, a eastern city of India, an annual mortality rate of 7.6 per 100,000 per year and a standardized mortality ratio of 2.58 was reported for epilepsy in a 5 -year follow-up of general population. ${ }^{1}$ Among the neurological causes, epilepsy was the second leading cause of death after stroke. There is a dearth of literature from India regarding deaths in PWE and the potential risk factors.

Another important domain in PWE is the risk of seizure-related injury (SRI). Features such as seizure-like impairment of consciousness and loss of motor control lead to a substantially increased risk of injury during ictus. PWE often have other comorbid conditions such as learning disabilities, and somatic and psychiatric conditions that 
place them at increased risk for accidental injury. Because of the increased risk of injury, PWE are subject to employment, recreational, and driving restrictions. These limitations have personal and social consequences, leading to inactivity, isolation, and dependency. Risk of injuries due to epilepsy is an important predictor of quality of life as it has a significant impact on an individual's social life, education, and employment status. Limitations in all these spheres of life may lead to a loss of independence, impaired social development, and increased psychiatric comorbidities.

We examined the rates and types of SRI based on self-reported questionnaires, and we also assessed the risk factors for SRI in a preregistered cohort of PWE attending an epilepsy clinic of a tertiary teaching hospital in Northern India. We also recorded deaths in the cohort and sought to find potential risk factors.

\section{Methods}

A total of 536 consecutive preregistered PWE were identified using a case survey in Delhi and the National Capital Region. PWE attending the epilepsy clinic of the Department of Neurology at the All India Institute of Medical Sciences in New Delhi, during the period from May 2010 to December 2011, were included in this ambispective cohort study. Inclusion criteria for the study were patients with active epilepsy of more than 1-year duration (the International League Against Epilepsy definition of active epilepsy is "at least one epileptic seizure in the previous 5 years, regardless of antiepileptic drug treatment"), and ability to read Hindi and give their informed consent to participate in the study. The purpose of the study was explained and informed consent was obtained from the patients or their caregivers. Structured questionnaires included demographic details such as age, gender, occupation, socioeconomic status, and personal habits along with birth and developmental history and mental status. Questionnaires were administered by a trained neurologist for all patients in either a personal or a telephone interview. Various factors were recorded, such as the age at onset of epilepsy, duration of disease, type and frequency of seizures, any premonition or aura, timing of seizures (nocturnal or daytime), control of epilepsy (controlled epilepsy is defined as either no seizure or less than 10 seizures per year and more than 10 seizures per year is defined as uncontrolled epilepsy), treatment details, and the probable etiology of epilepsy.

Patients were asked whether they had sustained any injury/injuries during a seizure in last 12 months. Injuries were classified as direct injury, such as orolingual trauma or tongue bite (due to the motor phenomenon of seizure) and indirect injuries such as consequence of a fall during a seizure (e.g., fracture or dislocation), burns, lacerations, soft tissue injuries, and dental injuries (needing medical attention). Participants were specifically asked about injuries like tongue bite, dental injuries, soft tissue injuries, head trauma, burns, orthopedic injuries, or seizures in water/drowning. Medical or surgical intervention received for the same were recorded. Documents pertaining to hospitalization and medical advice related to these injuries were also verified. The included participants were divided two groups: group 1 (patients who never sustained any SRIs) and group 2 (patients who had sustained an SRI).

Every 3 months, phone calls were made to inquire about the well-being of the participants. If a participant had died, details regarding the circumstances of their death were collected. The medical records of PWE who had died were also evaluated to collect details regarding the possible cause of death.

\section{Statistical analysis}

Data were presented as number (\%) or median (range), as appropriate. Continuous demographic and clinical variables of patients with and without SRI were analyzed using a Wilcoxon rank-sum test, and categorical variables were compared using chi-squared or Fischer's tests. To determine the factors associated with injury and death among PWE, logistic regression analysis was carried out. The results were presented as odds ratio (OR) with 95\% confidence intervals (Cls). Adjusted ORs were obtained and considered significant if the $\mathrm{Cl}$ did not exceed 1. A p-value of $<0.05$ was considered significant. Statistical analysis was carried out using STATA 9.0 (StataCorp LLC, College Station, TX, USA).

\section{Results}

A total of 526 patients with active epilepsy were enrolled in the study. Overall, 355 patients (67.5\%) had no SRIs whereas 171 patients (32.5\%) had sustained an SRI during the past 12 months. The demographic and clinical characteristics of both groups are tabulated in Table 1. There were significant differences between the two groups (SRI and no SRI) for the following parameters: gender, birth history, and mental status ( $p=0.01)$; seizures occurring while awake $(p=0.05)$, independence in daily activities $(p=0.007)$, uncontrolled seizures $(p=0.001)$, and use of more than two types of antiepileptic drugs ( $p=0.008)$. No significant difference was found between employed and unemployed participants and among those with differing 
Table 1. Distribution of cases, according to demographic data

\begin{tabular}{|c|c|c|c|}
\hline & $\begin{array}{l}\text { Group } 1 \text { (no injury) } \\
(\mathrm{n}=355,67.5 \%)\end{array}$ & $\begin{array}{c}\text { Group } 2 \text { (injury group) } \\
(\mathrm{n}=171,32.5 \%)\end{array}$ & $p$-value \\
\hline \multicolumn{4}{|l|}{ Gender } \\
\hline Male & $220(62)$ & $124(72.5)$ & \multirow{2}{*}{0.01} \\
\hline Female & $135(38)$ & $47(27.5)$ & \\
\hline \multicolumn{4}{|l|}{ Birth history } \\
\hline Normal & $346(97.4)$ & $159(93)$ & \multirow[t]{2}{*}{0.01} \\
\hline Abnormal & $9(2.6)$ & $12(7)$ & \\
\hline \multicolumn{4}{|l|}{ Mental status } \\
\hline Normal & $319(90)$ & $140(82)$ & \multirow[t]{2}{*}{0.01} \\
\hline Abnormal & $36(10)$ & $31(20)$ & \\
\hline \multicolumn{4}{|l|}{ Occupation } \\
\hline None/household work & $102(28.7)$ & $56(32.7)$ & \multirow{3}{*}{0.324} \\
\hline Laborer/high risk & $110(31)$ & $63(36.8)$ & \\
\hline Desk job & $133(37.5)$ & $50(29.2)$ & \\
\hline \multicolumn{4}{|c|}{ Socioeconomic status (modified Kuppuswamy scale) } \\
\hline Upper & $51(14.4)$ & $5(2.9)$ & \multirow{5}{*}{0.12} \\
\hline Upper middle & $35(9.9)$ & $26(15.2)$ & \\
\hline Lower middle & $52(14.6)$ & $32(18.7)$ & \\
\hline Upper lower & $104(29.3)$ & $34(19.9)$ & \\
\hline Lower & $113(31.9)$ & $74(43.3)$ & \\
\hline \multicolumn{4}{|l|}{ Care } \\
\hline Self-care & $328(92)$ & $145(85)$ & \multirow[t]{2}{*}{0.007} \\
\hline Caregiver & $27(8)$ & $26(15)$ & \\
\hline \multicolumn{4}{|l|}{ Seizure control } \\
\hline Controlled & $179(50)$ & $58(34)$ & \multirow[t]{2}{*}{0.001} \\
\hline Uncontrolled & $176(50)$ & $113(64)$ & \\
\hline \multicolumn{4}{|l|}{ Timing of seizure } \\
\hline Day & $323(90)$ & $146(85.4)$ & \multirow[t]{2}{*}{0.05} \\
\hline Night & $32(10)$ & $25(14.6)$ & \\
\hline \multicolumn{4}{|l|}{ Sensorium } \\
\hline Normal & $162(45.6)$ & $71(42)$ & \multirow[t]{2}{*}{0.374} \\
\hline Altered & $193(54.4)$ & $100(58)$ & \\
\hline \multicolumn{4}{|l|}{ Treatment compliance } \\
\hline Good & $341(96)$ & $159(93)$ & \multirow[t]{2}{*}{0.128} \\
\hline Poor & $14(4)$ & $12(7)$ & \\
\hline \multicolumn{4}{|l|}{ No. of antiepileptic drugs } \\
\hline 1 & $90(25.34)$ & $39(22.8)$ & \multirow[t]{3}{*}{0.008} \\
\hline 2 & $131(36.9)$ & $44(25.8)$ & \\
\hline 3 or more & $134(37.75)$ & $88(51.4)$ & \\
\hline
\end{tabular}

Values are presented as number (\%).

socioeconomic status ( $p=0.626$ and 0.703 , respectively).

Primary generalized tonic-clonicseizures (GTCS) occurred in 158 (30\%) patients, dyscognitive seizures in 100 (19\%), secondary generalization in 208 (39.4\%), focal non dyscognitive seizures in 21 (3.9\%), and myoclonic seizures in 39 (7.4\%) patients. In all, 129 patients were taking a single antiepileptic drug, 175 patients took two antiepileptic drugs, and 254 patients took three or more antiepileptic drugs. In addition to antiepileptic drugs, 32 patients (6\%) had undergone epilepsy surgery.

The most common etiologies based on radiologic parameters were hippocampal sclerosis (56 patients); central nervous system infection
(46 patients), of which 26 were neurocysticercosis; cortical dysplasia (13); developmental brain tumor (11); stroke-related causes (2), and other conditions (54). The latter included arteriovenous malformation, brainatrophy, hematoma, hypoxic brain damage, tuberous sclerosis, neuronal migration syndrome, and posttraumatic events.

\section{Types of injuries}

Soft tissue injury was the commonest type of SRI (60\%), with involvement of the face, lips, scalp, chin, arms, thigh, back, and buttocks. Suturing was required in only four patients. The second most common type of injury was tongue bite (52\%) followed by head 
30 Journal of Epilepsy Research Vol. 8, No. 1, 2018

Table 2. Risk factors for injury by multiple logistic regression analysis

\begin{tabular}{|c|c|c|c|c|}
\hline & \multicolumn{2}{|c|}{ OR $(95 \% \mathrm{Cl})$} & \multicolumn{2}{|c|}{ OR $(95 \% \mathrm{Cl})$} \\
\hline & Unadjusted & $p$-value & Adjusted & $p$-value \\
\hline \multicolumn{5}{|l|}{ Gender } \\
\hline Male & 1 & & & \\
\hline Female & $0.62(0.41,0.93)$ & 0.018 & $0.60(0.40,0.92)$ & 0.01 \\
\hline \multicolumn{5}{|l|}{ Birth history } \\
\hline Normal & 1 & & & \\
\hline Abnormal & $2.9(1.12,7.03)$ & 0.018 & $1.80(0.56,5.40)$ & 0.298 \\
\hline \multicolumn{5}{|l|}{ Mental status } \\
\hline Normal & 1 & & & \\
\hline Abnormal & $1.97(1.17,3.21)$ & 0.01 & $0.89(0.36,2.24)$ & 0.799 \\
\hline \multicolumn{5}{|l|}{ Occupation } \\
\hline Nil/household work & 1 & & & \\
\hline Laborer/high risk & $0.79(0.45,1.40)$ & 0.80 & & \\
\hline Desk job & $1.15(0.63,2.08)$ & 0.42 & & \\
\hline \multicolumn{5}{|l|}{ Care } \\
\hline Self & 1 & & & \\
\hline Caregiver & $2.18(1.23,3.86)$ & 0.008 & $1.5(0.56,4.08)$ & 0.425 \\
\hline \multicolumn{5}{|l|}{ No. of seizures (years) } \\
\hline Nil & 1 & & & \\
\hline$<10$ & $1.56(0.65,3.77)$ & 0.323 & & \\
\hline $10-20$ & $1.86(0.74,4.39)$ & 0.199 & & \\
\hline$>20$ & $4.14(1.72,9.11)$ & 0.002 & & \\
\hline \multicolumn{5}{|l|}{ Cluster/status epilepticus } \\
\hline Nil & 1 & & & \\
\hline Cluster/SE & $2.79(1.64,4.76)$ & 0.001 & $2.0(1.12,3.68)$ & 0.01 \\
\hline Duration of seizures & $1.01(0.99,1.0)$ & 0.154 & & \\
\hline \multicolumn{5}{|l|}{ Control of seizure } \\
\hline Controlled & 1 & & & \\
\hline Uncontrolled & $2.0(1.4,2.9)$ & 0.001 & $1.74(1.11,2.72)$ & 0.01 \\
\hline \multicolumn{5}{|l|}{ Timing of seizures } \\
\hline Day & 1 & & & \\
\hline Nocturnal & $1.7(1.0,3.0)$ & 0.06 & & \\
\hline \multicolumn{5}{|l|}{ Sensorium during seizures } \\
\hline Day & 1 & & & \\
\hline Nocturnal & $1.19(0.82,1.7)$ & 0.374 & & \\
\hline \multicolumn{5}{|l|}{ No. of antiepileptic drugs } \\
\hline 1 & 1 & & & \\
\hline 2 & $0.8(0.5,1.3)$ & 0.375 & $0.7(0.4,1.2)$ & 0.09 \\
\hline 3 or more & $1.5(0.96,2.45)$ & 0.08 & $0.94(0.60,1.62)$ & 0.81 \\
\hline \multicolumn{5}{|l|}{ Compliance to treatment } \\
\hline Compliant & 1 & & & \\
\hline Noncompliant & $1.84(0.83,4.07)$ & 0.133 & & \\
\hline \multicolumn{5}{|l|}{ Type of seizures } \\
\hline Generalized (primary \& secondary) & 1 & & & \\
\hline Focal seizures & $0.19(0.05-0.63)$ & 0.01 & $0.16(0.05,0.61)$ & 0.02 \\
\hline \multicolumn{5}{|l|}{ Type of seizures } \\
\hline Primary GTCS & 1 & & & 0.02 \\
\hline Dyscognitive seizures & $0.45(0.26,0.79)$ & 0.005 & $0.49(0.27,0.87)$ & 0.991 \\
\hline Myoclonic seizures & $0.80(0.39,1.16)$ & 0.550 & $0.91(0.46,12.16)$ & \\
\hline Focal seizures with secondary generalization & $0.65(0.43,1.0)$ & 0.05 & $0.60(0.39,0.97)$ & 0.04 \\
\hline Simple focal seizures -Non-dyscognitive & $0.24(0.068,0.85)$ & 0.02 & $0.23(0.06,0.84)$ & 0.03 \\
\hline
\end{tabular}

$p$-value $<0.05$ is significant.

$\mathrm{OR}$, odds ratio; $\mathrm{Cl}$, confidence interval; SE, status epilepticus; GTCS, generalized tonic-clonic seizures. 
trauma (20\%), which included concussion and head laceration. Two of our patients were hospitalized and hematoma evacuation was performed; one patient recovered completely and the other had residual weakness. Dental trauma occurred in $10 \%$ patients. Eight patients reported burns (5\%) that involved the face, foot, and whole body; in all these patients, burns occurred while cooking. One patient with whole-body burns was admitted to the hospital. Seventeen patients reported orthopedic injuries (10\%), three of these patients were treated with a plaster cast and patellectomy was performed in one patient.

\section{Activity during which injuries occurred}

The activity documented at the time of seizure were doing housework (43\%), working (24\%), sleeping (12\%), playing in school (11\%), traveling (5\%), and other (5\%).

\section{Risk factors for SRI}

After univariate analysis of potential risk factors for SRI, male gender ( $p=0.01)$, abnormal birth history $(p=0.01)$, abnormal mental status $(p=0.01)$, duration of epilepsy $(p=0.04)$, seizures occurring during the day $(p=0.05)$, dependence on a caregiver for carrying out activities $(p<0.008)$, uncontrolled seizures $(p=0.001)$, history of cluster seizures or status epilepticus $(p=0.001)$, and use of more than three types of antiepileptic drugs ( $p=0.008$ ) were found to be significant (Table 1). On multiple logistic regression analysis (Table 2), uncontrolled seizures (OR 1.74, 95\% Cl 1.11-2.72), history of cluster seizures or status epilepticus (OR 1.74, 95\% Cl 1.11-2.72), and occurrence of GTCS (primary or secondary) were found to be significant risk factors.

\section{Deaths and risk factors for death}

A total of 16 deaths were recorded in our cohort. In three patients, death was due to accident: two fell from the roof, presumably due to a seizure, and one drowned. Thirteen of these individuals died during a seizure, but none had status epilepticus. Of these 13 participants, four (30.7\%) had seizures while sleeping and died 15-30 minutes after a generalized seizure. The other nine (69.2\%) participants had presumed, but unwitnessed seizures; however, these were not in accident settings. There was a male predominance, with 10 out of 16 patients being male. On univariate analysis of potential risk factors for death, the only factor found to have statistical significance was uncontrolled seizures $(p=0.03)$.

\section{Discussion}

We conducted an ambispective cohort study of 526 preregistered PWE from a tertiary teaching hospital in North India. Overall, 33\% of respondents reported having sustained some form of SRI in their lifetime. Of these, only $5 \%$ required hospitalization for their injuries.

In a large prospective study by Beghi and Cornaggia ${ }^{2}$ of 951 patentsassessing the risk of accidents in PWE, 24\% had SRIs. In a questionnaire-based study by Sapna et al. ${ }^{3}$ from Southern India, $44.8 \%$ of respondents reported at least one injury in the previous 12 months. This proportion is much higher than the proportion in our series, which may be because those authors studied the frequency of both seizure-related and unrelated injuries in PWE.

The proportion of PWE who sustain SRI varies depending upon the study design and method of obtaining the figures. In a recent self- reported survey of 343 PWE from Australia, $55 \%$ of respondents reported to have SRI. In a retrospective population-based study of $247 \mathrm{PWE}$, followed up for 2,714 person-years conducted by Lawn et al. ${ }^{4} 16 \%$ of patients experienced at least one SRI. The most common type of injury in our study was soft tissue injuries (60\%). Many previous studies have also shown soft tissue injuries to be the most common type of injury among PWE. The risk of soft tissue injury has been reported to be as high as $74 \%$ in previous studies of adults and children. ${ }^{5}$

PWEs have a high risk for fractures. Fractures result from the interplay between accidental trauma, loss of protective reflexes during seizure and seizure-related falls, and associated osteoporosis due to long-term use of antiepileptic drugs. In a meta-analysis of the literature on fracture risk in epilepsy, it was found that PWE are twice as likely to sustain fracture as the general population. ${ }^{6}$ In our study, $10 \%$ of patients had dislocations and fractures of different bones.

In a population-based study of PWE by Téllez-Zenteno et al., ${ }^{7}$ a higher frequency of burns than that in the general population was reported. About $5 \%$ of participants in our study reported burns, all occurring while doing household work. This is lower than the rates reported in surveys conducted by Spitz et al. ${ }^{8}$ and Buck et al. ${ }^{9}$ The possible reason for the lower percentages of burns in our study could be due to differences in sociocultural practices in India. Joint families are the norm in Indian culture and PWE usually do not actively participate in cooking or other household chores that could expose them to a risk of burns.

Many factors have been implicated in increasing the risk of SRI. Of all the factors, type of seizure has the highest correlation with risk of SRI. In a retrospective population-based study by Lawn et al., ${ }^{4}$ uni- 
variate analyses identified five potential risk factors for SRI: greater number of antiepileptic drugs used, less independent living situation, higher Rankin score, history of generalized convulsive seizures or drop attacks, and higher seizure frequency score. Seizure frequency, however, was the only significant risk factor identified by multivariate analysis ( $p<0.001$; relative risk, 1.3). In agreement with their study, male gender, uncontrolled seizures, a history of cluster seizures or status epilepticus, and presence of GTCS were found to be significant risk factors in our series. In a questionnaire-based study of 300 patients from Thailand, the potential risk factors identified were occurrence of GTCS, seizures at least 12 times per year, and daytime seizures. ${ }^{10}$ In contrast to this, we found no significant correlation with the time of seizure and risk of SRI.

In a retrospective case-controlled cohort study using data from Danish registries, Holst et al. ${ }^{11}$ reported that having epilepsy increased the crude risk of death, with a hazard ratio of $11.9(95 \% \mathrm{Cl}$ 11.0-12.9). The findings of a community-based study from India among 37,844 inhabitants, including 173 PWE, suggested a higher risk of death in PWE than in the general population.

In our cohort of 526 PWE, 16 were reported to have died during seizures. Thirteen patients died unexpectedly and suddenly, without any evidence of status epilepticus or trauma; however, autopsy was not performed in any of these patients, which was mostly due to religious beliefs of the family members. These PWE fulfilled the criteria for probable SUDEP. One of the limitations of our study was that there was no control arm for comparison. Hesdorffer et al. ${ }^{12}$ analyzed pooled data from four case-control studies of risk factors for SUDEP, including 289 cases and 958 controls. Their findings indicated that people who have early-onset refractory symptomatic epilepsy with frequent GTCS and antiepileptic drug polytherapy are at higher risk of SUDEP. Our study found uncontrolled seizures to be the only factor associated with an increased risk of death.

Lamberts et al. ${ }^{13}$ reported that most of the SUDEP events were unwitnessed (86\%), and many were during sleep (58\%). In those with SUDEP, the seizure patterns were nocturnal in $36 \%$ and diurnal in $32 \%$ of individuals in that study. Showing a similar trend, $69.2 \%$ of our cases were unwitnessed and $30.7 \%$ were sleep related. Because of the sociocultural milieu in a country like India, discussing death is a taboo. Prospective case-controlled studies examining the potential risk factors for SUDEP would shed more light on this important aspect of epilepsy.

The limitations of our study include the lack of a control arm. There is a possibility of significant recall bias as the data were collected by administering a questionnaire to participant and not using medical records, thus decreasing the reliability of the information and with possible underreporting of mild SRIs. In addition, the study population was a hospital-based cohort of PWE; therefore, our results cannot be generalized to the general population.

In the present study, we found that male gender, occurrence of GTCS, uncontrolled seizures, and a history of cluster seizures or status epilepticus predicted the occurrence of injury in PWE. We found that uncontrolled seizures was the risk factor that could predict SUDEP. Precautions should be taken by caregivers of patients with these risk factors, to prevent injury.

\section{References}

1. Banerjee TK, Ray BK, Das SK, et al. A longitudinal study of epilepsy in Kolkata, India. Epilepsia 2010;51:2384-91.

2. Beghi E, Cornaggia C. Morbidity and accidents in patients with epilepsy: results of a European cohort study. Epilepsia 2002;43:1076-83.

3. Sapna CS, Sheeba SL, John J, Thomas SV. Accidents and injuries in people with epilepsy attending a tertiary care center in India. Epileptic Disord 2008;10:276-81.

4. Lawn ND, Bamlet WR, Radhakrishnan K, O'Brien PC, So EL. Injuries due to seizures in persons with epilepsy: a population-based study. Neurology 2004;63:1565-70.

5. Nakken KO, Lossius R. Seizure-related injuries in multihandicapped patients with therapy-resistant epilepsy. Epilepsia 1993;34:836-40.

6. Vestergaard P. Epilepsy, osteoporosis and fracture risk - a meta-analysis. ActaNeurolScand 2005;112:277-86.

7. Téllez-Zenteno JF, Hunter $G$, Wiebe $S$. Injuries in people with self-reported epilepsy: a population-based study. Epilepsia 2008;49:954-61.

8. Spitz MC, Towbin JA, Shantz D, Adler LE. Risk factors for burn as a consequence of seizures in persons with epilepsy. Epilepsia 1994;35:764-7.

9. Buck D, Baker GA, Jacoby A, Smith DF, Chadwick DW. Patients' experiences of injury as a result of epilepsy. Epilepsia 1997;38:439-44.

10. Tiamkao S, Sawanyawisuth $K$, Asawavichienjinda T, et al. Predictive risk factors of seizure-related injury in persons with epilepsy. J NeurolSci 2009;285:59-61.

11. Holst $A G$, Winkel BG, Risgaard B, et al. Epilepsy and risk of death and sudden unexpected death in the young: a nationwide study. Epilepsia 2013;54:1613-20.

12. Hesdorffer DC, Tomson T, Benn E, et al. Combined analysis of risk factors for SUDEP. Epilepsia 2011;52:1150-9.

13. Lamberts RJ, Thijs RD, Laffan A, Langan Y, Sander JW. Sudden unexpected death in epilepsy: people with nocturnal seizures may be at highest risk. Epilepsia 2012;53:253-7. 